\title{
How to Reach the Goal of Quadruple Aim Today in Healthcare Service Ecosystem: Nudges from the A4A Approach.
}

\author{
Nabil Georges Badr $^{1, *}$, Luca Carrubbo $^{2}$, Layal Mohtar ${ }^{1}$ \\ ${ }^{1}$ Higher Institute for Public Health, Université Saint-Joseph, Beirut \\ ${ }^{2}$ University of Salerno, Dept. of Business Science - Management \& Innovation Systems
}

\begin{abstract}
The pandemic has uncovered hidden, latent or unknown misalignments in healthcare worldwide. Difficulties, friction, disturbances, misunderstandings, underestimations, carelessness and other issues have ensued quickly, upsetting the ecosystem value creation mechanisms and influencing all actor's behavior within every country's Healthcare Service Ecosystems (H-SES). The expected health system impacts are those demarcated by the quadruple aims that include all the actors in the health ecosystem. Such aims address improving patient and caregiver experience; improving the health of populations; reducing per capita cost; and improving the work life of providers. This warranted a search for deeper understanding of the on-going dynamics and relative insights to foster actor engagement, provide a good global service in Healthcare, and fully reach the well-known quadruple aim. By referring to the cyclical model of the actor for actor model (A4A), we consider and review their shared intentionality and finality alignment, to investigate how resource integration work here and what happen in case of contingences (as in the case of a pandemic). It is a theoretical work, but entails many practical issues in Healthcare in terms of patient empowerment, improving population health, lowering cost of care, and maintaining the wellbeing of the care team.
\end{abstract}

Keywords. Healthcare Service Ecosystem, quadruple aims, A4A approach, pandemic era.

\section{Introduction}

The world was not prepared for disruptive events such as the COVID-19 pandemic, which caused a severe impact to the healthcare as intended service ecosystem (H-SES) $[1,2]$. That has highlighted the gaps in implementing a suitable strategy in terms of Value (value proposition, value perception, value co-creation), due to lack of precise information from population groups on their practice adherence (i.e. value-in-use) [3] to tailor new practices (i.e. value-in-experience) [4], and seek the engagement of all actors, clinical and non-clinical, in the specific context of local public and private healthcare services (i.e. value-in-context) [5]. In Healthcare, the existing link among value co-creation and system dynamics in the path of H-SES deeply matches with the 'continuity' of care service [6], as it is provided as

* Corresponding author: nabil@itvaluepartner.com 
something resulting from strong cooperation, great engagement, mutual benefit, and patientempowerment based healthcare plans and operations [7], following a lot the logic of win-win relationships as stated in Service Science and the studies of Smart Healthcare [8].

This allows having a specific focus on the main H-SES guidelines and final goals, like well-known quadruple aims evidenced in terms of improvement of patient experience, improved population health, reduction of cost of care, and a better work life of health care providers, including clinicians and staff [9].

Methodologically, to do that, the Actor-for-Actor (A4A) approach [10] has been used to frame the interactions and understand the constraints. By referring to the cyclical model of A4A steps, we focused on the structural pre-conditions of actor's engagement and relationships and reviewed the system determinants and their awareness in being part of an $\mathrm{H}-\mathrm{SES}$, their shared intentionality and finality alignment, to investigate how resource integration work here and what happen in case of contingencies - as in the case of a pandemic. This applied 'method theory' [11], by matching A4A main steps and healthcare quadruple aims - QAs (as compared in the fig.1), allowed us to adapt the lens used in confirming the current advances in knowledge and going toward practical suggestions for managers in Healthcare.
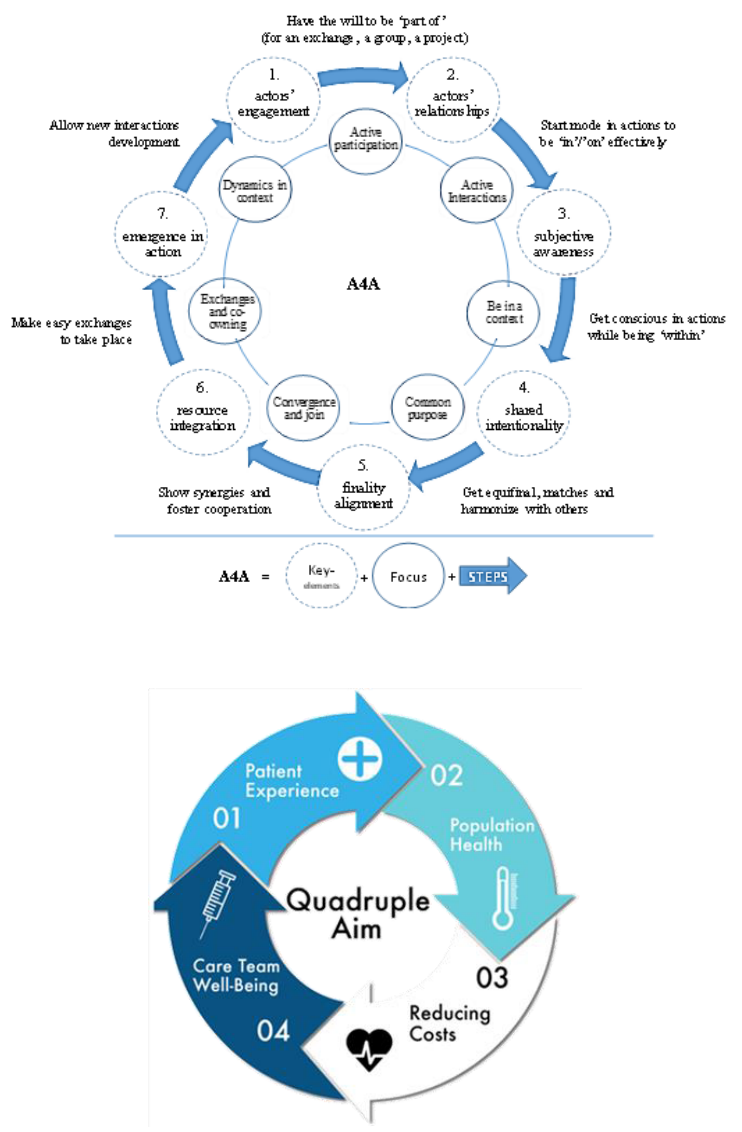

Fig.1. Crossing A4A approach left [10] and Healthcare Quadruple Aim [74] 


\section{Scientific frame based on systems' studies}

With the growing size of an aging population with chronic care conditions, it is imperative that public health, working with other healthcare organizations, use learning from COVID19 to work towards developing a client centric (patient or population group), service driven, community strategy to co-create and sustain value to public at large. The pandemic has uncovered hidden, latent or unknown misalignments in Healthcare worldwide; difficulties, friction, disturbances, misunderstandings, underestimations, carelessness and other issues have ensued quickly. This led to challenges in maintaining a continuity of care and the quality of service provision.

Systems' studies offer contextualized dynamic interactions among Actors, to frame the interpretation mode to be used here; then a focus on the H-SES has been done, by highlighting the typical switch from EGO to ECO logic [12]. It is interesting to understand which aspects are linked to sustainability, such as the multitude of actors (workers, citizens, producers, suppliers, authorities, consumers, users, etc.) and facilitators (retail sales, large distribution, networks information, financial services, public health administration), which are fundamental for the improvement of the management capacity and implementation of collaborative strategies [13]. In this sense, service systems (smart-, complex-, eco- that they are) do not derive from simple intuitions but result from systematic methods of continuous learning, data collection, rational innovation, social responsibility and network governance [14]. By applying an intelligent service to an intelligence in practice, inserted in a context of smart cities, made up of intelligent organizations, through intelligent operations, to achieve intelligent results, there can be some important changes in every aspect of our daily life [15]. As regards the definition of the reference framework, it seems useful to appropriately consolidate the level of analysis. The study of complex phenomena, often framed in broader discourses than single events, in fact requires a precise investigation perspective that is capable of enhancing the contributions represented up to now by the reference scientific paradigms $[16,17]$.

\subsection{Capability Reconfiguration}

Capability reconfiguration processes follow interpretative logics aimed at reducing complexity, new information architectures are favored and infrastructures must strengthen organizations, computing and system performance, allowing for better management of complexity [18] The variety of information and the variability of possible reticular interconnections within the service systems promote new forms of co-operation that can be interpreted as systemic interactions between the actors of the cognitively aligned system [19]. The system in this sense is made viable by the behavior assumed, more strategic, more reactive, more adaptive, more intelligent [20]. The qualification and enhancement of these interactions, the redesign of organizational configurations, the management of complexity are therefore all elements that identify a successful system [21]; furthermore, given the service-oriented perspective of modern operations, such a structured service system, which exploits network synergies can in effect be considered smart, able to really survive within its own eco-system of reference [18]. When environmental contingencies occur (contingency theory), organizations are able to survive in a particular context only if they improve their ability to evolve and make their operations adhere to external changes [22]. In fact, the opening of the systems studied (service systems for SSMED and SD Logic, value networks for network theories, viable systems for VSA), involves a homeostatically dynamic adaptation to external changes and their survival is directly linked to ability to seek and promote dynamic and satisfactory evolutions (equifinality) [23]. While the world is getting smarter (we are now talking more and more often about smarter planet), systems must be 
people-centric, information-driven, e-oriented to adapt, and mutual and collective satisfaction should encourage and cultivate people to collaborate and innovate [24].

By analyzing the Healthcare context from a system perspective, it is possible to define all the Actors as endogenous elements of a single and interactive value generation process. Everyone therefore has (or should have) an active role, is dynamic and decisive for the evaluation of the service rendered as a whole, even in the health sector. According to some fundamental principles of Service Science, the interactions between these Actors can be classified as follows [25, 26]. In an H-SES, several stakeholders (patients, suppliers, health organizations, authorities) participate in the exchange process in a vision which sees the logic of service as crucial [27]. There is a heterogeneity of actors (patients, clinicians, administrators, authorities and others) who are part of the health system as bearers of very different interests, which therefore bring equally different resources to the system [28]. They have undergone a series of changes that have changed their principles, objectives, aims and logic of operation.

\subsection{Patient Centricity and Collaborative Value co-creation}

In particular, the re-organization of healthcare is aimed at placing the citizen at the center of the system and its planning, trying to make him not simply aware of the care process and of the reasons for which company choices are made, but also participate in the process of creation of value through the desire to share information with other patients, the collection and correct management of data and information useful for the purposes of the medical history and traceability of the diagnostic or therapeutic path, self-medication, periodic medical checks and other behaviors estimates and proactive activities [29]. The attempt is being made to provide the tools through which citizens can dialogue, intervene in the care process, and promote requests for improvement. However, the effectiveness of this process is strictly connected with the effective interaction and cooperation between the various subjects and resources involved, which is possible only through the implementation of coordination mechanisms of different types, i.e. at the managerial, political, social, economic level and IT [30]. Ultimately, the modern process of using the health service could be defined as a "cooperative game", aimed at the promotion, implementation and coordination of multiactor interventions [31].

According to the studies on Systems, however, the (objective) environment, in which the (subjective) context of each specific system is developed and consolidated, is able to affect the daily strategies undertaken and pursued, enhancing or debasing the attitudes and the relative survival capacities (for example of plants, but also of companies and organizations in general) in the long term of all systems (more or less rooted, more or less contextualized) [17]. To survive, organizations cannot fail to relate to their own subjective reference context, as well as respect the constraints and rules of the over-determined objective environment [32]. For a given system, the environment is therefore that set of exchange objects whose characteristics affect and are influenced by the behavior of a system [17]. The various approaches deriving from the general theory of systems precisely define the environment/context relationship, with particular reference to the influence exerted on systems and their behaviors [17].

More in depth, the "viable model of systems" deriving from VSM outlines the concept of a vital system, as an entity that is adaptable to its constantly evolving environment and therefore able to survive [33]. The viable system is an abstract description of cybernetics, applicable to all autonomous organizations. From it derives the study of the structure of the regulations, of the reactions to changes, of the feedbacks, that is to say of all that allows the adaptation of the system to the new conditions of the surrounding environment [34]. 
As far as healthcare organizations are concerned, the service they are required to provide, in favor of collective health and its sustainability, becomes a real corporate vision [8]. All the various stakeholders interact with the social context, government agencies, ethics and legal issues in order to improve the proposed / perceived average level of quality [15]. Behaviors and decisions to adapt have a very specific place in a complex environment such as healthcare, and for this reason they deserve to be analyzed [7]. The healthcare environment, made up of infinite subjective contexts, is in fact a function of the different diagnostic paths, the different knowledge possessed, the different resources available, all aspects that generate different scenarios, but which at the same time fall within the same general framework represented by the Healthcare, seen as a Service Ecosystem (H-SES) as in the following section.

\section{Observing the Healthcare Service Ecosystem}

"In a well-functioning health care system, the creation of value for patients should determine the rewards for all other actors in the system" [34]. Value assessment in general is to be seen from a consumer's view-point in service driven economies, especially it is all about how healthcare consumers are impacted by the spread of viral infection in a pandemic. Public health 3.0 research argues that public health agencies have to re-envisions themselves to become population ecosystem centric by developing community strategies that leverage cross-sector collaboration of actors within the community to address the distinct needs of various population groups [35]. This calls for a learning health system approach that assess how environmental, policy, and system level actions will influence the social determinants of health of individual population groups and tailor practices to influence their health behavior, exactly as in a H-SES. To do this, we often try to propose new tools, practices, measures, communication channels, connections; we adapt, we learn, we change, to strengthen ourselves and be more competitive over time [36].

Graphically (fig.2) it is possible to observe how the patients more than the other Actors are able to interact with all the others, can obtain benefit from these interactions, can contribute (through appropriate feedback, coded or not) to support the provision of the service itself and indirectly to improve the quality of performances (in terms of efficiency and effectiveness) [7].

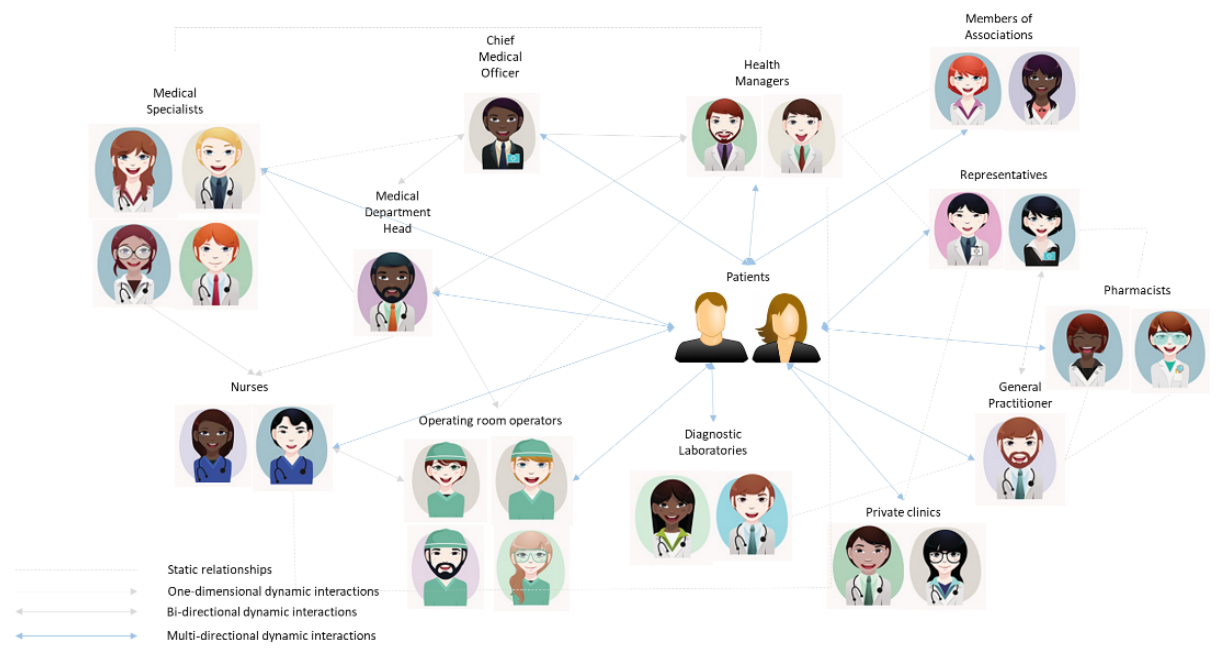

Fig.2. Configuring today multi-level HSES [7] 
The links between the entities operating in the H-SES carry the links relating to the procedures indicated by the institutions that each actor (clinics, pharmacies, industries) must comply with, as in practice we see every day. In the same way, the flow of information (concerning rules, constraints, norms, limits) improves the relationships between the individuals who operate and who are served. In the health sector, the eco-systemic essence leads to a safer diagnosis, clinical knowledge has increased, hospitals can therefore be more efficient and can really contribute to improving sustainable collective well-being [7].

\section{The contribution of $A 4 A$ in reaching quadruple aims in healthcare}

A4A model is a set of actor-to-actor relationships [10, 37]. These 'A4A' type relationships, mutual interest of subjects, are defined over a given process and oriented to seek mutual satisfaction among the actors, affecting a lot the concept of value and stressing multi-level co-creative processes involving actors as in the H-SES. These are to lead to long-term relationships, thus leaving little room for opportunistic and/or speculative behaviour.

The engagement can potentially lead to co-creation of value and willingness to commit resources to support the shared goals. In the healthcare, culture plays a role in how clients interact with clinical care providers and the language used and empathy shown can go a long way in seeking commitment to a shared outcome, and potentially influencing adherence to treatment guidelines or preventive practices.

\subsection{Applying the A4A lens}

In $\mathrm{A} 4 \mathrm{~A}$, the commitment does not only focus on generic psychological involvement of the actors, but also identifies the actors' intention to share in a common purpose that is pursued in a specific way to enable them to participate towards the common purpose. When there is a high sensitivity to collective realization, the alignment of strategies in the planning phase increases, as does their viability $[38,39,40]$. To discuss A4A application in-detail we use its seven steps [10] and apply main reflections on nowadays-healthcare evolving situations:

\subsubsection{Actors' engagement}

Step 1 (actors' engagement) begins with actors become interested in a given situation and need to play specific roles and be involved and 'engaged' in a given relationship. This becomes a part of this engagement. For example, in the smoking cessation program, a patient playing the role as a parent, may include the support of family members, physician and staff to engage in a specific relationship that gets all involved, in order to sustain the health behaviour. Engagement of family members and community has become more common way to extend the H-SES to sustain health behaviour such as improve health literacy and address food insecurity.

\subsubsection{Actors' relationships}

Once the initial 'connection' of relevant actors has led to begin the engagement, it is possible to witness continuous interactions, activated by the harmony in both the goals and common purpose among all involved as in the step n.2 (actors' relationships). In fact, lots organizations in H-SES recognize the need for collective engagement in addressing food insecurities and improving health literacy, there are pilot efforts underway to begin the engagement process and revise and refine the goals. 


\subsubsection{Subjective awareness}

When the context within which this engagement is populated by multiple actors, whose behaviour can directly or indirectly influence (on the other hand in a reciprocal way) the engagement, then this awareness becomes a part of recognizing various spheres of culture that can influence the success of such an engagement as a whole [32, 33, 34] as shown in the step n.3 (subjective awareness). For example, during Covid-19, the engagement of new actors became necessary in addressing the needs of clients such as social and community health workers and technology providers and they bring a different culture to the engagement.

\subsubsection{Shared intentionality}

Recognizing the cultural differences that influence each agent should lead to nurturing these so that there is empathy, trust and encouragement of all involvement to stay focused on the shared goals and harmony in purpose highlighted in step n.4 (shared intentionality). For example, healthcare organizations' staff recognized the differing competencies among community health workers, who are tasked to provide healthcare screening and education using health monitors and digital apps, and developed culturally sensitive information and easy to use technologies to support the shared goal of sustain health screening. This allowed to focus on other client needs such as testing and addressing food and economic insecurities.

\subsubsection{Finality alignment}

When the cooperation of all involved comes with a conviction that their working together can lead to mutual satisfaction (actual or latent), it allows them to work towards the collective goals and overcome gaps of the step n.5 (finality alignment). For example, in some cases, healthcare managers recognized during the first value cycle that health literacy [41] that focused on nutrition and physical activity during Covid-19 is not sufficient as clients needed support to address their mental health issues. This led to engaging a new educator of mental health in the health literacy program, so as to ensure that all are working to address the evolving needs of the clients on mental health during Covid-19.

\subsubsection{Resource integration}

This positive condition of such actor engagement becomes the foundation for a continued exchange of information and sharing of resources deemed necessary to support sustained value in spite of evolving changes in the ecosystem. This becomes critical as a part of a longer-term strategy of step n.6 (resource integration), as minor adaptations due to changing ecosystem demands should not distract the engagement of actors to support the shared goals that has started the initial interaction [37].

\subsubsection{Emergence in action}

Finally, by operating in this type of continually evolving dynamic, a virtuous circuit of value cycles are used to remain agile in spite of changes in both customer and organizational culture [39] as evidenced in step n.7 (emergence in action). For example, today many hospitals continue to explore the role of remote monitoring technologies to enable clients to selfmanage their health and engage social service providers in improving access to care among underserved neighborhoods [35]. This process will continue with appropriate agreement of the desired health outcomes and monitoring these outcomes to make the multi-actor engagement agile to empower clients and improve their health behavior [39]. 


\subsection{Elaborating the quadruple aim of the H-SES under the A4A lens}

A4A focuses precisely on the motivational drivers using a systems' dynamics approach that includes pro-activity, the convergence of ideas and values, shared intentionality, equality, cognitive alignment, integration of resources, emergency and viability [10]. There are systemic elements and structural elements that deserve investigation. The A4A logic occurs when there is [10]

- Active participation with a reciprocal contribution in other people's activities;

- Active interactions: being in context, conscious of action and outcome; empathy takes on a role;

- Common purpose and shared intentionality with a mutual interest in a shared action, in non-opportunistic and non-speculative behaviours, growing alignment of strategies in the programming phase

- Convergence through synergies and cooperation, with a high sensitivity to collective satisfaction; The network logic and the System effect (positive sum) are clearly evident from the dynamic interchange between the actors of the supply chain;

- Exchange and co-ownership, with a certain awareness and a clear will on the part of the subjects involved; the value is inevitably co-generated, the contribution is multipart; the very viability of an organization is commensurate with the realization of the propensity for change (including structural).

- Dynamics in Context - allowing new interactions to develop with a commitment and self-involvement are the basis of every interaction, to facilitate the co-creation process as much as possible, organizations tend to adapt by constantly reconfiguring;

Therefore, A4A approach would be a compass of optimizing health system performance, through the adaptation of the quadruple aim to enhance patient experience, improve population health, reduce costs, and improve the work life of healthcare providers, including clinicians and staff. The systems perspective, described above, allows to identify the critical elements, including environmental ones, with which it must confront, in order to cope with the numerous external and internal changes, which, especially in the long term, can influence its work [42]. As we continue this section, we elaborate this A4A lens to the quadruple aim of the H-SES. This approach and the outcome of our work, could be helpful in facilitating service continuity and adaptation of Actors and under pressure in today's complex H-SES. This can help us in matching A4A main steps and healthcare quadruple aims - QAs (as compared in the fig.1), as follows:

\subsubsection{Better patient experience}

To enhance patient experience (QA-1), actors are engaged (A4A-1), building relationships, learning, and knowledge sharing while interacting (A4A-2). The concept of learning is fundamental above all for so-called "intelligent" systems, which may be able to selfreconfigure in order to resist / persist in a sustainable way over time. The very concept of "smart" (a term recently proposed and accredited in the field of research on the Service) implies well-defined characteristics for the systems; systems are defined as intelligent because they react to external changes through the rational and intelligent use of available resources, adapting their structure to the use of knowledge, trying to "feel" their context and acquiring / selecting new useful experiences. In these cases, the supply chain is reconceptualized as a network of service systems and for this reason it presents a configuration that cannot be defined a priori, but rather capable of being iridescent, adapting and evolving with respect to the changing conditions of the context. The contribution of knowledge, the application of skills, the ability to configure and re-configure, the desire to establish longterm relationships with subjects considered strategic all represent elements of a systemic way 
of being adaptive. Learnings from the pandemic inform future practices. Knowledge sharing among service providers in the H-SES offer opportunities leading to greater resilience in the healthcare system [44]. Data, collected and transformed into tacit knowledge through actor collaboration [45], can support decision makers in addressing the surrounding complexity by making the H-SES more smart and resilient and promoting its renewal [46]. For example, the pandemic has given swift rise to online communities among older adults' communal coping practices [47]. These knowledge platforms helped shape the learnings and increased the outreach to an otherwise quiet actor population [48]. Elsewhere, the pandemic has pushed the boundary of virtual care. Technologies such as telehealth [49] and care at home [50] have improved access to specialty care. Ultimately, with reference to healthcare, smart logics produce significant effects in terms of efficiency, quality of service [51], user satisfaction, highlighting the comparison between the different real intelligent service systems and the evaluation of relative performance: each smart service systems is able to improve its solutions and achieve sustainable success, thus becoming a viable system! Data-driven decisions drive the H-SES well-being only when they imply an immediate improvement of the patients' experience in the care of their health.

\subsubsection{Improved population health}

To improve population health (QA-2), actors strive towards awareness of their context (A4A3 ) as they align (A4A-4) to a common purpose (A4A-5). A fundamental step in healthcare management is therefore to develop an intelligent organization that bases its management and strategies on advanced technological solutions within and outside an H-SES. Innovations in technology that integrate actors from the value cycle of care delivery contribute to the adherence to clinical management guideline [52], and the satisfaction of both patients and providers [53]. Different actors such as payers, providers and carers must understand societal values such as patient preference and willingness-to-pay, in order to quantify the value of new interventions and extra-clinical outcomes associated effective treatments [54]. The decrease in mortality rates in ICUs through uniform transparency about care and referral protocols [52] and reduction of sepsis risks [55] can be witness of such actor alignment to decrease length of stay within progressive care units, availing the services to more patients and contribution to an overall better population health outcome [56]. The healthcare global service ought to organize in a functional way to maximize its usefulness for the different needs of users and the availability of resources of the actors involved in the delivery process. Management coordination is required and therefore the introduction of strong integration mechanisms to allow functioning in a new, contemporary, more interactive and collaborative logic, in order to achieve concrete results, and at the same time to favor innovative processes aimed at identifying solutions originals for the benefit of the user first.

\subsubsection{Reduced cost of care}

As they aim for cost reduction and resource democratization (QA-3), Actors prioritize resource integration (A4A-6). While value co-creation within the service exchange is perceived through the sensitivity of the actors involved, proper information and knowledge management contribute to building awareness that drives resource integration for the benefit of the H-SES as a whole [10]. The integration of these direct and indirect actors, with their activities and interactions, contributes to enabling the value proposition of the service [57]. Resource integration is necessary to co-create value and develop new interactions. For example, during the pandemic emergency, the need for social protection and social distancing provided incentive for innovation, and telemedicine has emerged as an efficient and effective way to provide care while reducing hospital overload and COVID-19 spread, care costs have 
decreased on average of $30 \%$ per claim [49]. Such resource integration initiatives spawned new end-to-end innovation adoption models that require the combination of technology, practice and legislative resources [58]. Elsewhere, chronic disease management, faced with competing priorities for service provisioning, drove the evolution of the H-SES through enhanced coordination and resource integration between different actors (patients, doctors, service providers, relatives, caregivers, etc.). Coordination between actors in the supply chain, wearable device manufacturers and drug makers delivered virtual adherence aids, care at home technologies [59] and monitoring mechanisms to reduce the risk on patients and lower the cost of care [60]. In this sense, the functionality of healthcare service systems does not derive from intuition or chance, but from systematic methods, continuous learning, timely data collection, rational innovation, social responsibility and network governance.

\subsubsection{Improving the wellbeing of the care team}

Actors learn to adapt and reconfigure to the new dynamics of service continuity (A4A-7) to improve the work-life of the providers (QA4) - restarting the dynamic cycle of the quadruple aim. Through adaptation, actors realize the effective contextual conditions for directing their efforts toward shared benefits derived from a shared purpose [61]. Hospitals have adapted technology systems and operational processes to improve efficiencies and provide timelier access to specialty care [49] and decreased emergency department utilization [62], reflecting on lesser workload on the staff. The quality of the nursing practice has improved by adapting resources and relationships to different modalities of care [63], improving the morale affecting workforce engagement and safety such as workforce burn-out [64] as structural, technological and systemic adaptations are formalized [65]. This leads to a spirit of constant adaptation (structural and systemic) and therefore is the result of a continuous and continuously stimulated reasoning, encouraging more interaction, co-creation, eternalizing the A4A cycle.

\section{Reflections through the virtuous example of telemedicine}

Central to the reconfiguration capability of the H-SES, health technology focuses on the interaction between the patient and the multiple actors and services in the healthcare ecosystem. Healthcare technology are tools available to healthcare professionals aimed at improving not only the quality of care, but also the production efficiency of the healthcare macro-sector. Thanks to emerging technology in healthcare, such as telemedicine, the disruption of the service delivery due to the pandemic has attenuated.

Telemedicine is therefore a great example of this value proposition through our adapted lens of the A4A/QAIM:

- Actors' engagement - relationships, learning, and knowledge sharing: Through the application of telemedicine technology, actor engagement has increased, enabled through non-conventional interactions between the multiple actors in the H-SES. For instance, telemedicine using smartphones have enabled more flexible and mobile work [66] with emphasis on continuity of care at home [67].

- Actors pursue awareness of context as they align to a common purpose: All actors in this contexts were assured a continuous value cycle, aligning to a common purpose. The practice of telemedicine has improved patient experience through ensuring timeliness of care $[62,63,49]$ and better access $[68,69,50]$, while leading to an improved quality of life for care seekers [70] and care givers [53]. Telehealth use has been connected to decreased hospitalization and resource utilization [62], lessening the workload on the care staff [71], thus improving the work life of health care providers [65] and reducing cost of care [72,73] while improving the outcome of care [54]. 
- Actors prioritize resource integration and democratization: Hospitals adopting telehealth have seen improvement in operational efficiencies and timelier access to specialty care [49]. For example, timely diagnosis, with the use of telehealth for minor illnesses in children and adolescents decreased emergency department utilization by $22 \%$ [60]. All the same, telehealth programs can be cost saving for intermediate and high-risk patients over a 1 - to 5-year window [73]. The cost reduction can be direct through actual cost of intervention or consultation and indirect through reduction in the consumption of hospital services [72].

- Actors learn to adapt and reconfigure - dynamics of Continuity: With the implementation of telehealth in the model of care, satisfaction levels at the provider experience have improved [71]. Provider satisfaction have improved in $94 \%$ of cases of telehealth implementations [65], an indication that actors were able to adapt and reconfigure. Telehealth consultations contribute to the adherence to clinical management guideline [52] and the satisfaction of both patients and providers [53] and showed no significant difference in adherence to treatment guidelines across the multiple care modalities (primary care, urgent care, etc.) of which telemedicine has become one.

\section{Conclusions and the limitations/future research insights}

Summarizing, the premise of the A4A approach presents the notion that actors engage, establish relationships, each in their subjective awareness, aligning their objectives to the equifinality of the outcome, while they integrate the system's resource in an evolving value cycle [10]. Through this lens, the concept of continuous adaptation translates into fundamentals of actor engagement, serving the essential aims of H-SES (Figure 3).

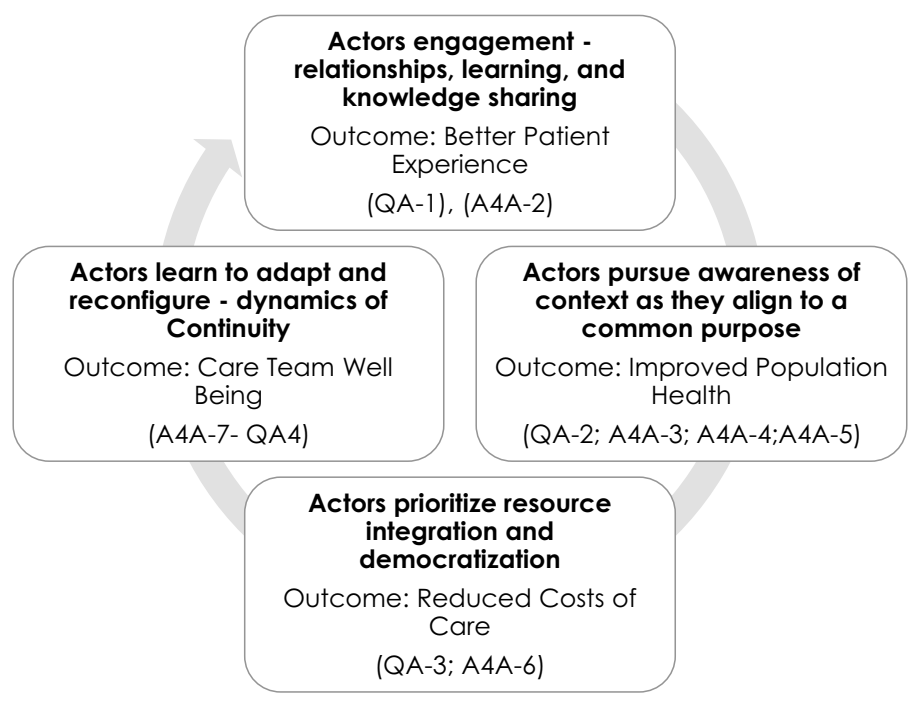

Fig.3. Quadruple Aim through the lens of A4A for actor engagement - A4A/QAIM

The sharing of purpose, the integration of resources, systemic equality and knowledge sharing are the synthesis of the relationship "4" (or "for" logic). In view of A4A, in every dynamic system the equilibrium once reached is maintained with repeated behaviors, which in a certain sense constitute the historical memory of the interaction, and which do not cancel 
each other out on the occasion of the modifications that may occur in the meantime; an interactive system does not start from scratch every time, but maintains the conquests acquired even when it has to look for other balances.

Actor engagement fosters value creating relationships, continuous learning, and knowledge sharing, leading to better patient experience. As actors pursue deeper awareness of their context (such as a pandemic), they align to a common purpose, to improve population health. Actors prioritize resource integration and democratization for the reduction of cost of care and they learn to adapt and reconfigure while navigating the dynamics of value provisioning. This adaptation capability contributes to a sense of wellbeing. The A4A model can then be a compass for optimizing health system performance, through the adaptation of the quadruple aim, which could be helpful in overcoming actual problems and mistakes by giving some nudges for today's H-SES.

Therefore, our paper informs the practice of Healthcare management in the complex HSES. It proposes actionable knowledge grounded on theory, with a real contribution to Service Science through the suggestion of interaction evolution in service innovation roadmaps that exploit the learning systems imbedded in these interactions [75] while identifying specific value creating outcomes in alignment with the quadruple aims. Nevertheless, further research is encouraged to use our proposed alignment as cornerstone to investigate the multiple facets of the interaction, considering digital actors, and other nonhuman consitutuents of the complex healthcare ecosystem.

\section{References}

1. Frow P, McColl-Kennedy JR, Hilton T, Davidson A, Payne A, Brozovic D (2014). Value propositions: A service ecosystems perspective. Marketing Theory 14(3):327351.

2. Frow, P., McColl-Kennedy, J. R., \& Payne, A. (2016). Co-creation practices: Their role in shaping a health care ecosystem. Industrial Marketing Management, 56, 24-39.

3. Ballantyne, D., \& Varey, R. J. (2006). Creating value-in-use through marketing interaction: the exchange logic of relating, communicating and knowing. Marketing theory, 6(3), 335-348.

4. Sandström, S., Edvardsson, B., Kristensson, P., \& Magnusson, P. (2008). Value in use through service experience. Managing Service Quality: An International Journal.

5. Chandler, J. D., \& Vargo, S. L. (2011). Contextualization and value-in-context: How context frames exchange. Marketing theory, 11(1), 35-49.

6. Badr, N. G., Carrubbo, L., \& Ruberto, M. (2021). Responding to COVID-19: Potential Hospital-at-Home Solutions to Re-configure the Healthcare Service Ecosystem. In HEALTHINF, 344-351.

7. Polese, F., \& Carrubbo, L. (2017). Eco-sistemi di servizio in sanità (Vol. 65). G Giappichelli Editore.

8. Carrubbo, L., Clarizia, F., Hysa, X., \& Bilotta, A. (2013). New'smarter'solutions for the healthcare complex service system. Gummesson, E., Mele, C., Polese, F.(a cura di), System Theory and Service Science: Integrating Three Perspectives In a New Service Agenda, Giannini, Napoli, 14-17.

9. Badr, N. G., Chami, E., \& Asmar, M. K. (2021). e-Health Implementation in Lebanese Teaching Hospitals: What Can We Learn from Their Successes and Imminent Challenges. In HEALTHINF (pp. 304-311).

10. Polese, F., Pels, J., Tronvoll, B., Bruni, R., \& Carrubbo, L. (2017). A4A relationships. Journal of Service Theory and Practice. 
11. Jaakkola, E. (2020). Designing conceptual articles: four approaches. AMS review, 10(1), 18-26.

12. Barile, S., Carrubbo, L., Iandolo, F., \& Caputo, F. (2013). From'EGO'to'ECO'in B2B relationships. jbm-Journal of Business Market Management, 6(4), 228-253.

13. Capra F.(1997), The Web of Life, Flamingo, London.

14. Spohrer, J. C., Demirkan, H., \& Krishna, V. (2011). Service and science. In The Science of Service Systems (pp. 325-358). Springer, Boston, MA.

15. Watzlawick, P., \& Sanders, J. (1976). La realtà della realtà: comunicazione disinformazione confusione. Astrolabio.

16. Von Bertalanffy, L. (1968). General System Theory. George Braziller. Inc., New York, 84.

17. Hall, A.D., Fagen, R.E. (1956), "Definition of System", in General Systems (Yearbook of the Society for the Advancement of General Systems Theory), (1), 18-28.

18. Demirkan, H., \& Goul, M. (2006). AMCIS 2006 panel summary: Towards the service oriented enterprise vision: Bridging industry and academics. Communications of the Association for Information Systems, 18(1), 26.

19. Checkland, P. B. (1981). Systems thinking, systems practice John Wiley \& Sons. New York.

20. Espejo R., Harnden R.J. (1989), The Viable System Model, John Wiley, London.

21. Waldrop, M. M. (1993). Complexity: The emerging science at the edge of order and chaos. Simon and Schuster.

22. Beer, S. (1972), Brain of the Firm, The Penguin Press, London.

23. Barile, S., Saviano, M., Polese, F., \& Di Nauta, P. (2012). Reflections on service systems boundaries: A viable systems perspective: The case of the London Borough of Sutton. European Management Journal, 30(5), 451-465.

24. Qiu, R. G. (2009). Computational thinking of service systems: Dynamics and adaptiveness modeling. Service Science, 1(1), 42-55.

25. IBM (2009), Patient centered medical home. Cambridge, UK: University of Cambridge Institute for Manufacturing.

26. IBM (2012), The value of building sustainable health system. Cambridge, UK: University of Cambridge Institute for Manufacturing.

27. Chakraborty, S., \& Dobrzykowski, D. D. (2013). Supply chain practices for complexity in healthcare: A service-dominant logic view. IUP Journal of Supply Chain Management, 10(3).

28. Von Foerster, H. (1981). Observing Systems Intersystems. Seaside, CA.

29. Begun, J. W., Zimmerman, B., \& Dooley, K. (2003). Health care organizations as complex adaptive systems. Advances in health care organization theory, 253, 288.

30. Avison, D., \& Young, T. (2007). Time to rethink health care and ICT?. Communications of the ACM, 50(6), 69-74.

31. IBM (2012), The value of building sustainable health system. Cambridge, UK: University of Cambridge Institute for Manufacturing

32. Parsons, T. (1971). The system of modern societies.

33. Saviano, M., Bassano, C., \& Calabrese, M. (2010). A VSA-SS approach to healthcare service systems the triple target of efficiency, effectiveness and sustainability. Service Science, 2(1-2), 41-61. 
34. Porter, M. E. (2010). What is value in health care. N Engl J Med, 363(26), 2477-2481.

35. DeSalvo, K. B., O’Carroll, P. W., Koo, D., Auerbach, J. M., \& Monroe, J. A. (2016). Public health 3.0: time for an upgrade. American journal of public health, 106(4), 621.

36. Barile, S., Polese, F. \& Carrubbo, L. (2012), "Il Cambiamento quale Fattore Strategico per la Sopravvivenza delle Organizzazioni Imprenditoriali", in Barile, S., Polese, F., Saviano, M.(eds), Immaginare l'innovazione, e-book Giappichelli Ed., Torino, 2-32.

37. Polese, F., Carrubbo, L., Caputo, F., \& Megaro, A. (2018, September). Co-creation in action: An acid test of smart service systems viability. In International Conference on Exploring Service Science (pp. 151-164). Springer, Cham.

38. Barile, S., Polese, F., \& Carrubbo, L. (2012). Il cambiamento quale fattore strategico per la sopravvivenza delle organizzazion imprenditoriali (Change as strategic leverage for organizations' survival). Barile, S., Polese, F., Carrubbo, L.(2012), "Il Cambiamento quale Fattore Strategico per la Sopravvivenza delle Organizzazioni Imprenditoriali”, in Barile, S., Polese, F., Saviano, M.(a cura di), Immaginare l'innovazione, e-book Giappichelli Editore, Torino, 2-32.

39. Barile, S., Pels, J., Polese, F., \& Saviano, M. (2012). An introduction to the viable systems approach and its contribution to marketing. Journal of Business Market Management, 5(2), 54-78.

40. Barile, S., Polese, F., Saviano, M., Carrubbo, L., \& Clarizia, F. (2012). Service Research Contribution for Health Networks' Understanding. Innovative Service Perspectives, Hanken School of Economics, 71.

41. Tanniru, M. R., Agarwal, N., Sokan, A., \& Hariri, S. (2021). An Agile Digital Platform to Support Population Health-A Case Study of a Digital Platform to Support Patients with Delirium Using IoT, NLP, and AI. International journal of environmental research and public health, 18(11), 5686.

42. Polese, F., Carrubbo, L., Bruni, R., \& Maione, G. (2017). The viable system perspective of actors in eco-systems. The TQM Journal.

43. Authors' elaboration on known models under discussion.

44. Brodie, R. J., Ranjan, K. R., Verreynne, M. L., Jiang, Y., \& Previte, J. (2021). Coronavirus crisis and health care: learning from a service ecosystem perspective. Journal of Service Theory and Practice.

45. Badr, N. G., Dragoicea, M., \& Crihana, I. (2020, October). What Do We Know About Renewable Knowledge and Sustainable Societal Growth? A Scoping Review. In 17th conference of the Italian Chapter of AIS (Association for Information Systems).

46. Carrubbo, L., Megaro, A., \& Notari, F. (2021, April). Data-Driven Decisions for a Smarter and Resilient Healthcare Service Ecosystem. In The International Research \& Innovation Forum (pp. 69-77). Springer, Cham.

47. Aghdam, A. R., Watson, J., Miah, S. J., \& Cliff, C. (2021). A novel information sharing framework for people living with type-2 diabetes in the context of a group education program. Health Information Science and Systems, 9(1), 1-16.

48. Kamalpour, M., Eden, R., Syed, R. A., Buys, L., Tariq, A., \& Watson, J. (2021). What older adults do in online communities when they co-create and co-destruct value. International Journal of Quality and Service Sciences.

49. Lum, H. D., Nearing, K., Pimentel, C. B., Levy, C. R., \& Hung, W. W. (2020). Anywhere to anywhere: use of telehealth to increase health care access for older, rural veterans. Public Policy \& Aging Report, 30(1), 12-18. 
50. Lavin, B., Dormond, C., Scantlebury, M. H., Frouin, P. Y., \& Brodie, M. J. (2020). Bridging the healthcare gap: Building the case for epilepsy virtual clinics in the current healthcare environment. Epilepsy \& Behavior, 111, 107262.

51. Badr N. G., Mohtar, L (2021) Telehealth: A Viable Option for Optimizing Health System Performance during COVID-19 - Call to Action for Future Pandemics. HealthINF 2022.

52. Steinman, M., Morbeck, R. A., Pires, P. V., Abreu Filho, C. A. C., Andrade, A. H. V., Terra, J. C. C., ... \& Kanamura, A. H. (2015). Impact of telemedicine in hospital culture and its consequences on quality of care and safety. Einstein (Sao Paulo), 13(4), 580586.

53. Waibel, K. H., Bickel, R. A., \& Brown, T. (2019). Outcomes from a regional synchronous tele-allergy service. The Journal of Allergy and Clinical Immunology: In Practice, 7(3), 1017-1021.

54. Snoswell, C., Smith, A. C., Scuffham, P. A., \& Whitty, J. A. (2017). Economic evaluation strategies in telehealth: obtaining a more holistic valuation of telehealth interventions. Journal of telemedicine and telecare, 23(9), 792-796.

55. DeJong, C., Santa, J., \& Dudley, R. A. (2014). Websites that offer care over the Internet: is there an access quality tradeoff?. JAMA, 311(13), 1287-1288.

56. Armaignac, D. L., Saxena, A., Rubens, M., Valle, C. A., Williams, L. S., Veledar, E., \& Gidel, L. T. (2018). Impact of Telemedicine on Mortality, Length of Stay, and Cost Among Patients in Progressive Care Units: Experience From a Large Healthcare System. Critical care medicine, 46(5), 728-735.

57. Vargo, S.L., Koskela-Huotari, K., Edvardsson, B., Baron, S., Reynoso J., Colurcio, M. (2017), A Systems Perspective on Markets: Building a Research Agenda, Journal of Business Research,79, pp.260-268.

58. Guarcello, C., \& Raupp, E. (2021). Pandemic and innovation in healthcare: The end-toend innovation adoption model. BAR-Brazilian Administration Review, 18.

59. Badr, N. G., Carrubbo, L., \& Ruberto, M. (2021). Responding to COVID-19: Potential Hospital-at-Home Solutions to Re-configure the Healthcare Service Ecosystem.

60. Sebastiani, R., \& Anzivino, A. (2021). Transformative Value Co-creation in Healthcare Services in the COVID-19 Era: The Case of Centro Cardiologico Monzino. The Future of Service Post-COVID-19 Pandemic, Volume 1, 55.

61. Lee, J., \& Han, S. H. (2021). Preparing for Accelerated Third Order Impacts of Digital Technology in Post Pandemic Service Industry: Steep Transformation and Metamorphosis. The Future of Service Post-COVID-19 Pandemic, Volume 1, 1., T., Kapros, E. (2020). Co-creation and co-design in technology-enhanced learning: Innovating science learning outside the classroom. Interaction Design and Architecture (s), 42, pp.202-226.

62. Gattu, R., Teshome, G., \& Lichenstein, R. Telemedicine Applications for the Pediatric Emergency Medicine. Pediatric Emergency Care, 32(2), 123-130 (2016).

63. Caffery, L. J., Farjian, M., \& Smith, A. C. (2016). Telehealth interventions for reducing waiting lists and waiting times for specialist outpatient services: A scoping review. Journal of telemedicine and telecare, 22(8), 504-512.

64. Sikka, R., Morath, J. M., \& Leape, L. (2015). The quadruple aim: care, health, cost and meaning in work. 
65. Lopo, C., Razak, A., Maidin, A., Rivai, F., Mallongi, A., \& Sesa, E. (2020). Technology impact on healthcare quality of the hospital: A literature review. Enfermería Clínica, 30, 81-86.

66. Tuckson, R. V., Edmunds, M., \& Hodgkins, M. L. Telehealth. New England Journal of Medicine, 377(16), 1585-1592 (2017).

67. Nakagawa, K., \& Yellowlees, P. M. (2019). University of California Technology Wellness Index: a physician-centered framework to assess technologies' impact on physician well-being. Psychiatric Clinics, 42(4), 669-681.

68. Qureshi, Ijaz \& Raza, Hassan \& Whitty, Michael \& Naqvi, Zain.Telemedicine implementation and benefits for quality and patient safety in Pakistan. Knowledge Management \& E-Learning: An International Journal, 367-377 (2015).

69. Lurie, N., \& Carr, B. G. (2018). The role of telehealth in the medical response to disasters. JAMA internal medicine, 178(6), 745-746.

70. Waibel, K. H., Cain, S. M., Hall, T. E., \& Keen, R. S. (2017). Multispecialty synchronous telehealth utilization and patient satisfaction within Regional Health Command Europe: a readiness and recapture system for health. Military medicine, 182(7), e1693-e1697.

71. Bashir, A., \& Bastola, D. R. (2018). Perspectives of nurses toward telehealth efficacy and quality of health care: pilot study. JMIR medical informatics, 6(2), e35.

72. Mehrotra, A., Paone, S., Martich, G. D., Albert, S. M., \& Shevchik, G. J. A Comparison of Care at E-visits and Physician Office Visits for Sinusitis and Urinary Tract Infection. JAMA Internal Medicine, 173(1), 72 (2013).

73. Liu, S. X., Xiang, R., Lagor, C., Liu, N., \& Sullivan, K. Economic Modeling of Heart Failure Telehealth Programs: When Do They Become Cost Saving? International Journal of Telemedicine and Applications, 2016, 1-9 (2016).

74. Bodenheimer, T., \& Sinsky, C. (2014). From triple to quadruple aim: care of the patient requires care of the provider. The Annals of Family Medicine, 12(6), 573-576.

75. Spohrer, J. (2021). Service innovation roadmaps and responsible entities learning. In ITM Web of Conferences (Vol. 38, p. 01001). EDP Sciences 